\title{
The Differences in the Epidemiology and Predictors of Death between Candidemia Acquired in Intensive Care Units and Other Hospital Settings
}

\author{
Chen $\mathrm{Li}^{1}$, Hao Wang ${ }^{1}$, Mei Yin ${ }^{2}$, Hui Han ${ }^{1}$, Jin-Feng Yue ${ }^{1}$, Fan Zhang ${ }^{1}$, \\ Ti-Chao Shan ${ }^{1}$, Hai-Peng Guo ${ }^{1}$ and Da-Wei $\mathrm{Wu}^{1}$
}

\begin{abstract}
Objective The burden of candidemia is shifting from intensive care units (ICU) to non-ICU settings. This study aimed to define the differences in epidemiology and predictors of death between ICU-acquired candidemia (ICUAC) and non-ICUAC.

Methods We conducted a retrospective study of 80 patients with ICUAC and 147 patients with non-IUCAC at five hospitals.

Results The distribution of Candida species and resistance to antifungal agents did not differ between the ICUAC and non-ICUAC groups. ICUAC patients received more echinocandins and less triazoles, as well as more adequate antifungal therapy than non-ICUAC patients (all $\mathrm{p}<0.05$ ). ICUAC patients had a significantly higher average acute physiology and chronic health evaluation (APACHE) II score (21.0 \pm 7.9 vs. 17.8 \pm 8.6 ; $\mathrm{p}<0.01)$, Sequential Organ Failure Assessment score $(9.2 \pm 5.5$ vs. $7.4 \pm 3.9 ; \mathrm{p}<0.05)$ and day-90 mortality rate $(52.5 \%$ vs. $36.7 \%$; $\mathrm{p}<0.05)$ when compared to non-ICUAC patients. Using a multivariate logistic analysis, adequate antifungal therapy was found to be the only protective factor for death in both groups. Respiratory failure supported with invasive mechanical ventilation, renal failure supported with replacement therapy and an APACHE II score $\geq 20$ were independent predictors of death in ICUAC patients, while age $\geq 60$ years, concurrent bacteremia and APACHE II score $\geq 20$ were independent predictors of death in non-ICUAC patients. Conclusion The Candida species and antifungal resistance profiles in patients with ICUAC were similar to non-ICUAC patients, but led to worse outcomes. The protective and risk factors for death may therefore be relevant for the clinical management of patients with candidemia in ICU and non-ICU settings.
\end{abstract}

Key words: candidemia, epidemiology, predictors, death, intensive care unit

(Intern Med 54: 3009-3016, 2015)

(DOI: 10.2169/internalmedicine.54.3744)

\section{Introduction}

Candidemia has emerged as an important nosocomial infection associated with significant morbidity and mortality $(1,2)$. It is the fourth most common nosocomial bloodstream infection in the United States and the seventh in Europe $(3,4)$. In intensive care units (ICUs), candidemia is a serious problem, and an estimated $33 \%$ to $55 \%$ of all episodes occur in ICUs $(5,6)$. However, the incidence of can- didemia has increased over the last decade in non-ICU populations, and the burden of candidemia is shifting from ICUs to general hospital wards (7-9). A number of studies from different regions have provided endemic epidemiology data regarding candidemia in ICU populations or other atrisk populations (10-15), but comparisons between ICUacquired candidemia (ICUAC) and non-ICUAC in a single region remain scarce.

The predictors of death in patients with candidemia are important for the implementation of early intervention. Re-

${ }^{1}$ Department of Critical Care Medicine, Qilu Hospital, Shandong University, China and ${ }^{2}$ Department of Geriatrics, Qilu Hospital, Shandong University, China

Received for publication July 24, 2014; Accepted for publication March 25, 2015

Correspondence to Dr. Da-Wei Wu, shandongicu@gmail.com 
cent studies have disclosed that various risk factors, including an ICU stay, older age, a higher acute physiology and chronic health evaluation (APACHE) II score, neutropenia, corticosteroid therapy (16-18) and inadequate or lack of initial antifungal treatment $(18,19)$, are factors associated with candidemia-related death. However, it is unknown whether these risk factors independently predict poor outcomes both in the patients with ICUAC and patients with non-ICUAC.

In this study, we retrospectively examined differences in the epidemiology and risk factors for death in patients with ICUAC and non-ICUAC at multiple centers in a city in China. We aimed to provide information for ICU and nonICU physicians to help direct anti-Candida therapy and improve the prognosis of candidemia.

\section{Materials and Methods}

\section{Study design and patient selection}

We performed a multi-center retrospective study of candidemia during hospital admission in five tri-service general hospitals in Jinan, China: Qilu Hospital of Shandong University (2,000 beds), Jinan Military General Hospital (2,000 beds), Qianfoshan Hospital affiliated with Shandong University (1,500 beds), Jinan Center Hospital affiliated with Shandong University (1,000 beds) and Shandong Provincial Hospital (Jinan, 2,000 beds). Data on patients admitted from January 2008 to June 2010 were reviewed. We included patients older than 16 years who had ICUAC or non-ICUAC. Candidemia was defined as at least one positive blood culture for Candida species in patients hospitalized for more than 48 hours with signs or symptoms of infection. ICUAC represented candidemia acquired more than 48 hours after ICU admission and non-ICUAC candidemia represented candidemia acquired more than 48 hours after admission to non-ICU settings. All episodes of candidemia were identified via the laboratory computer system. Post-discharge survival data were obtained from the Follow-up Departments of the hospitals. For patients with multiple candidemic episodes, only the first episode was included. Patients with candidemia caused simultaneously by different species of Candida were excluded. The study was approved by the Ethics Committee of Qilu Hospital of Shandong University and carried out with the ethical standards set forth in the Helsinki Declaration of 1975.

\section{Data collection}

Trained study team members collected demographic and clinical data by chart review. Demographic and microbiological data, predisposing factors, laboratory data, antifungal agent exposure and outcome were recorded on standardized case report forms. Underlying diseases, including solid organ tumor, hematologic malignancy, neutropenia, diabetes mellitus and chronic renal failure, were recorded. Predisposing factors that occurred within 30 days before the onset of candidemia were assessed. At the onset of candidemia (within two days following collection of the first positive blood sample), laboratory data were collected and the severity of the initial presentation of candidemia was assessed by APACHE II (20) and Sequential Organ Failure Assessment (SOFA) scores (21). We recorded the antifungal agents used for more than two days within the window of two weeks before through two weeks after the microbiological documentation of candidemia infection.

During the study period, there was no change of policy in collecting Candida strains. Candida species were identified by use of the VITEK-32 system (BioMerieux Vitek, St. Louis, USA). Minimum Inhibitory Concentrations (MIC) of fluconazole, voriconazole, itraconazole and amphotericin B were determined using the ATB Fungus 3 microbroth method (BioMerieux, La Balme-les-Grottes, France). The procedure was performed according to the manufacturer's guidelines. All resistant isolates were confirmed using Etest $^{\circledR}$ (AB Biodisk, Solna, Sweden). We used the recently updated Clinical and Laboratory Standard Institute (CLSI) species-specific antifungal drug susceptibility thresholds for Candida after incubation at $35^{\circ} \mathrm{C}$ for 24 hours (22-25). Regarding fluconazole, isolates of $C$. albicans, C. tropicalis and $C$. parapsilosis with MICs of $\geq$ eight $\mu \mathrm{g} / \mathrm{mL}$ and isolates of $C$. glabrata with MICs of $\geq 64 \mu \mathrm{g} / \mathrm{mL}$ were considered resistant. Isolates of $C$. krusei were considered intrinsically resistant to fluconazole. For voriconazole, isolates of C. albicans, C. tropicalis and C. parapsilosis with MICs of $\geq$ one $\mu \mathrm{g} / \mathrm{mL}$ and $C$. krusei isolates with MICs of $\geq$ two $\mu \mathrm{g} /$ $\mathrm{mL}$ were considered resistant. Susceptibility results for itraconazole were categorized according to interpretive breakpoints recommended by the CLSI (25). Since no breakpoints have been published by CLSI for amphotericin B, isolates inhibited by $>$ two $\mu \mathrm{g} / \mathrm{mL}$ were considered as resistant (26).

Neutropenia was defined as an absolute neutrophil count $<0.5 \times 10^{9} / \mathrm{L}$. Immunosuppressive drugs received included glucocorticoids ( $\geq 20 \mathrm{mg}$ /day of prednisone or equivalent doses of other corticosteroids for more than one week), chemotherapy drugs or other immunosuppressive agents. Septic shock was defined as systolic blood pressure $<90 \mathrm{mmHg}$, diastolic blood pressure $<60 \mathrm{mmHg}$ or if fluid/inotropes were required to maintain blood pressure above these levels (27). Patients treated with adequate antifungal therapy were defined as those who met all of the following criteria: (i) antifungal agent with in vitro activity against the Candida species causing the infection; (ii) the administration of an antifungal agent with an adequate dosage according to the Infectious Diseases Society of America (IDSA) guidelines (28); e.g., the initial adequate dosage of fluconazole for susceptible isolates was defined as six $\mathrm{mg} / \mathrm{kg} /$ day for $C$. albicans, $C$. tropicalis and $C$. parapsilosis in the face of normal renal function, and three $\mathrm{mg} / \mathrm{kg} / \mathrm{day}$ if creatinine clearance was $<50 \mathrm{~mL} / \mathrm{min}$. Fluconazole was not considered to be adequate at any dosage for C. krusei or C. glabrata; (iii) antifungal administration for more than three days; (iv) administration of antifungal treatment within 72 hours after drawing the first positive blood sample for culture. 


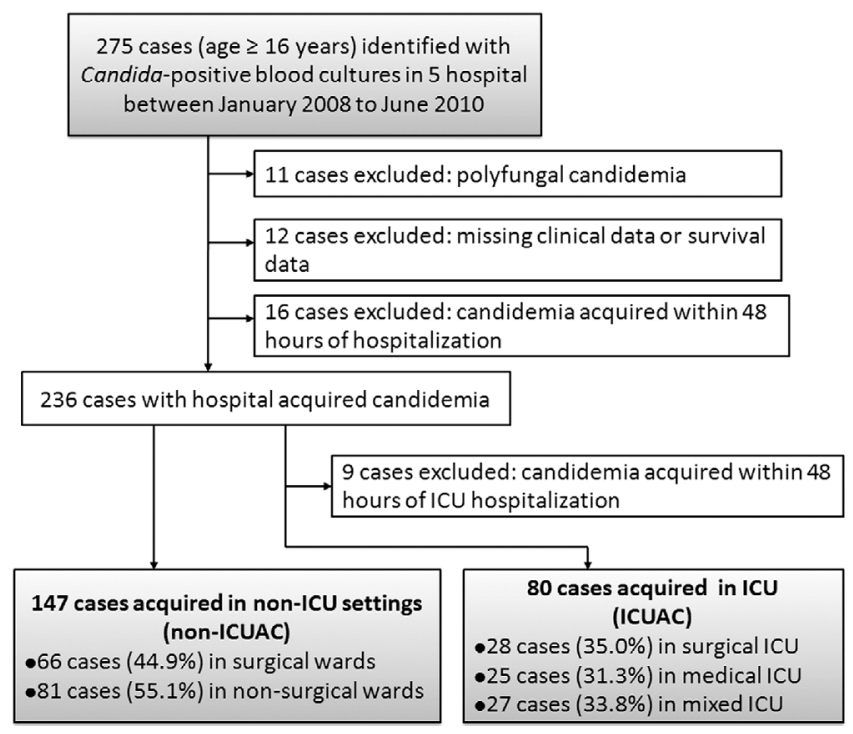

Figure 1. Flow diagram of inclusion criteria for cases with Candida-positive blood cultures. ICU: intensive care unit, ICUAC: intensive care unit acquired candidemia

\section{Statistical analysis}

The data for categorical variables were expressed as a percentage and continuous variables were expressed as the mean $\pm \mathrm{SD}$ or the median (interquartile range). A Chisquare or Fisher's exact test (two-tailed) was used to compare categorical variables and unpaired Student's $t$ test to compare continuous variables. The Kaplan-Meier analysis and log-rank test were used to compare day-90 survival. Multivariate, backwards, stepwise, logistic regression analyses were used to identify independent risk factors associated with day-90 mortality of patients with ICUAC and nonICUAC; the results were presented as odds ratios (ORs) with $95 \%$ confidence intervals (95\% CIs) and $\mathrm{p}$ values. Statistical analysis was performed using the SPSS 15.0 for Windows software package (SPSS, Chicago, USA). A p value of $<0.05$ was considered statistically significant.

\section{Results}

\section{Patient demographics, epidemiology and risk factors for candidemia}

A total of 275 cases were identified with Candidapositive blood cultures. Of these, 227 cases of candidemia in 227 patients were included in the analysis (Fig. 1). Eighty candidemia cases were acquired in an ICU (ICUAC, 35.2\%) and 147 cases were acquired in non-ICU settings (nonICUAC, 64.8\%), with an incidence of 15.1 cases/10,000 admissions and 1.65 cases/10,000 admissions, respectively. In both groups, $C$. albicans was the most commonly isolated species, followed by $C$. tropicalis and C. parapsilosis. The proportion of different Candida species and the resistance rate to antifungal agents did not differ between the ICUAC and non-ICUAC groups (all $\mathrm{p}>0.05$, Table 1 ).
Table 1. Species Distribution and Resistance to Antifungal Agents of Candida Isolated from Patients.

\begin{tabular}{|c|c|c|c|}
\hline & $\begin{array}{l}\text { ICUAC } \\
(\mathrm{n}=80)\end{array}$ & $\begin{array}{c}\text { Non-ICUAC } \\
(\mathrm{n}=147)\end{array}$ & $\mathrm{p}$ value \\
\hline \multicolumn{4}{|l|}{ Candida Species } \\
\hline Candida albicans & $32(40.0)$ & $63(42.9)$ & 0.677 \\
\hline Candida tropicalis & $18(22.5)$ & $35(23.8)$ & 0.824 \\
\hline Candida parapsilosis & $12(15.0)$ & $21(14.3)$ & 0.884 \\
\hline Candida glabrata & $8(10.0)$ & $18(12.2)$ & 0.612 \\
\hline Candida krusei & $9(11.3)$ & $7(4.8)$ & 0.068 \\
\hline Other & $1(1.3)^{*}$ & $3(2.0)^{\dagger}$ & 0.924 \\
\hline \multicolumn{4}{|c|}{ Resistance to antifungal agents } \\
\hline Fluconazole & $15(18.8)$ & $22(15.0)$ & 0.461 \\
\hline Itraconazole & $11(13.8)$ & $14(9.5)$ & 0.331 \\
\hline Voriconazole & $8(10.0)$ & $11(7.5)$ & 0.513 \\
\hline Amphotericin B & $2(2.5)$ & $3(2.0)$ & 0.804 \\
\hline \multicolumn{4}{|l|}{ Data are presented as $\mathrm{n}(\%)$. } \\
\hline
\end{tabular}

When compared with non-ICUAC patients, ICUAC patients had greater age, more often had underlying chronic renal insufficiency, more often received total parenteral nutrition, central venous catheter (CVC) or urethral catheter placement, and more often underwent respiratory failure supported with invasive mechanical ventilation and renal failure supported with renal replacement therapy (all $\mathrm{p}<0.05$; Table 2). The incidence rates of solid tumor, hematological malignancy and neutropenia were greater for non-ICUAC than ICUAC cases (all $\mathrm{p}<0.05$; Table 2).

\section{Antifungal therapies, laboratory data and outcomes of patients}

When compared to non-ICUAC patients, patients with ICUAC more often received echinocandins $(28.8 \%$ vs. $6.1 \%$; $\mathrm{p}<0.001)$ and less often received triazoles $(67.5 \%$ vs. $81.0 \%$; $\mathrm{p}=0.023$ ). ICUAC patients also received more adequate antifungal therapy than non-ICUAC patients $(47.5 \%$ vs. $38.8 \%$; $\mathrm{p}=0.044$; Table 3).

When compared with non-ICUAC patients, ICUAC patients had significantly lower platelet counts and higher levels of serum creatine, blood glucose and serum sodium and potassium, and a significantly higher APACHE II score $(21.0 \pm 7.9$ vs. $17.8 \pm 8.6 ; \mathrm{p}=0.006)$ and SOFA score $(9.2 \pm 5.5$ vs. $7.4 \pm 3.9 ; \mathrm{p}=0.010$ ). ICUAC patients also displayed a significantly higher day-30 mortality rate $(35.0 \%$ vs. $22.4 \%$; $\mathrm{p}=$ $0.042)$ and day- 90 mortality rate $(52.5 \%$ vs. $36.7 \%$; $\mathrm{p}=$ $0.022)$ and had significantly lower survival at 90 days (logrank test: $\mathrm{p}=0.016$; Table 3 and Fig. 2).

\section{Protective and risk factors for death in the two groups}

Using a univariate analysis, the protective and risk factors for day-90 mortality with statistical significance were determined in the ICUAC and non-ICUAC groups (Table 4, 5). Using a multivariate analysis, adequate antifungal therapy was found to be the only protective factor for death in both the ICUAC and non-ICUAC groups (OR 0.5 with $95 \% \mathrm{CI}$ 


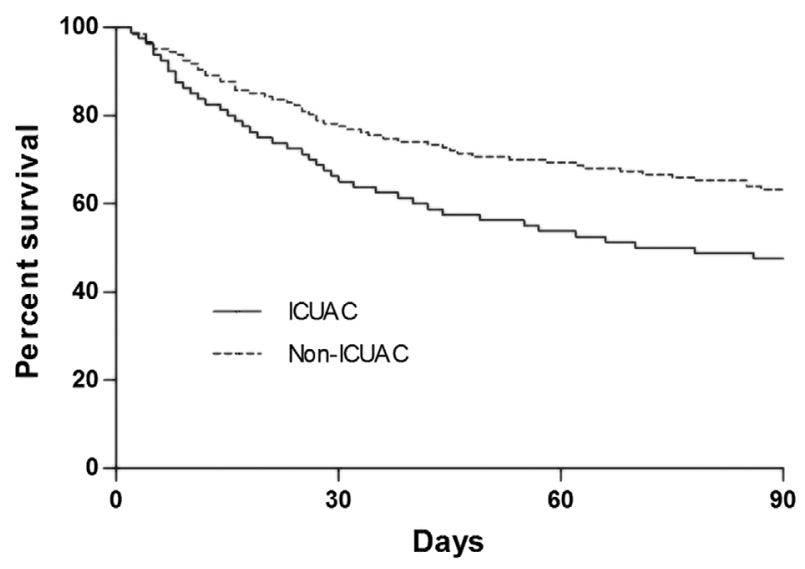

Figure 2. Kaplan-Meier survival analysis at day 90 for patients with ICUAC and non-ICUAC. ICUAC: intensive care unit acquired candidemia. $p$ value according to log-rank test is 0.016 .

ent risk factors for death in ICUAC patients, while an age $\geq 60$ years old (OR 5.4; 95\% CI 1.2-33.6; $\mathrm{p}=0.015$ ) and concurrent bacteremia (OR 2.7; 95\% CI 1.3-15.8; $\mathrm{p}=0.032$ ) were independent risk factors for death in non-ICUAC patients.

\section{Discussion}

In this study, a higher incidence of candidemia was observed in ICU than in non-ICU settings. However, the distribution of Candida species and the resistance to antifungal agents were similar between the ICUAC and non-ICUAC groups. The administration of adequate antifungal therapy was found to be the only protective factor for death in both groups. ICUAC patients with severe organ failure needed supportive implementation, while non-ICUAC patients with advanced age and concurrent bacteremia had significantly higher risk for death. The predictors of death may be relevant for the clinical management of patients with candidemia in ICU and non-ICU settings.

The different Candida species may vary in their susceptibility to various antifungal agents (29). Thus, a better understanding of the epidemiology of candidemia in the ICU and other hospital settings is important for clinicians to implement prophylactic or empirical therapy. According to recent studies, the distribution of Candida species differs by hospital units. C. albicans dominates in hemato-oncology units while non-albicans Candida occurs frequently in General Surgery units and in ICUs (30). Similarly, the incidence rate of $C$. tropicalis and C. krusei infection differs between internal medicine wards, surgical wards and ICU wards (31). In this study, however, the distribution of Candida species and resistance to antifungal agents were similar between ICU and non-ICU settings. The same results were reported by the SENTRY Antimicrobial Surveillance Program (32). Therefore, for ICU physicians, local pathogen characteristics in non-ICU settings can also be referenced when choosing an- tifungal agents.

We identified several healthcare-related factors associated with ICUAC, including multiple blood product transfusion, mechanical ventilation and CVC and urethral catheter placement, suggesting that candidemia might be preventable by a reduction in unnecessary medical procedures whenever feasible in ICU settings. In a previous report (33), removal of a CVC was associated with decreased mortality. Considering that CVC placement is more common in patients with ICUAC than in patients with non-ICUAC, the management and early removal of an unnecessary $\mathrm{CVC}$ requires more attention in the ICU. The CVC removal rate in this study was only $48.3 \%$ (data not shown), indicating that the awareness of the risk of CVC retention needs to be strengthened in the management of candidemia. In addition, although candidemia is associated with high mortality, reported mortality rates range from 38 to $85 \%$ among studies that included different patient populations (34-36). In this study, it is not surprising that ICUAC patients had poorer outcomes when compared to non-ICUAC patients, since they were much more severe, as reflected by a higher incidence of septic shock and higher APACHE II and SOFA scores.

A delay in the initiation of antifungal therapy in hospitalized patients with candidemia has been shown to significantly impact mortality (37). In this study, delayed therapy was included as a component of inadequate antifungal therapy, which occurs commonly and results in substantial prolongation of the hospital length of stay and increased hospital costs (38). Efforts to enhance the rates of appropriate therapy for candidemia may improve resource use. Adequate treatment was associated with reduced mortality (39), and was found to be the only protective factor in both groups of this study, indicating that standard antifungal therapies administered according to accepted guidelines should be encouraged in both the ICU and non-ICU settings. In this study, the rate of "adequate antifungal therapy" was quite low $(41.9 \%)$ and the main reason for this finding might be related to the lack of knowledge with regard to the IDSA guidelines of candidemia treatment (28) among the physicians in the participating hospitals.

The different predictors of death may be relevant for differential clinical management of patients with candidemia in ICU and non-ICU settings. Severe organ failure is the main cause of death in ICU patients, and even patients receiving organ supportive implementation are still at high risk of death $(40,41)$. In this study, the predictors of death in ICUAC patients included several organ supportive implementations, indicating early correction of organ failure and removal of these supportive implementations might improve the prognosis of ICU patients with candidemia. Interestingly, these supportive implementations were not predictors of death in non-ICUAC patients. Instead, the underlying host conditions, including advanced age and concurrent bacteremia, were found to be predictive for death in non-ICUAC patients, highlighting that prompt initiation of adequate antifungal therapy is especially important in the candidemic pa- 
Table 4. Univariate Analysis of Outcome in Patients with ICUAC.

\begin{tabular}{lccc}
\hline Characteristics & $\begin{array}{c}\text { Non-survived } \\
(\mathrm{n}=42)\end{array}$ & $\begin{array}{c}\text { Survived } \\
(\mathrm{n}=38)\end{array}$ & $\mathrm{p}$ value \\
\hline Age $\geq 60$ years & $26(61.9 \%)$ & $14(36.8 \%)$ & 0.025 \\
Chronic renal insufficiency & $15(35.7 \%)$ & $5(13.2 \%)$ & 0.020 \\
Respiratory failure supported with invasive mechanical ventilation & $33(78.6 \%)$ & $18(47.4 \%)$ & 0.004 \\
Renal failure supported with renal replacement therapy & $16(38.1 \%)$ & $2(5.3 \%)$ & 0.001 \\
Antifungal therapy started before microbiological documentation & $14(33.3 \%)$ & $22(57.9 \%)$ & 0.027 \\
Echinocandins usage & $8(19.0 \%)$ & $15(39.5 \%)$ & 0.044 \\
Adequate antifungal therapy & $13(31.0 \%)$ & $25(65.8 \%)$ & 0.002 \\
Platelet count $\leq 100 \times 10^{9} / \mathrm{L}$ & $14(33.3 \%)$ & $5(13.2 \%)$ & 0.034 \\
Serum creatine $\geq 180 \mu \mathrm{mol} / \mathrm{L}$ & $17(40.5 \%)$ & $7(18.4 \%)$ & 0.032 \\
Total bilirubin $\geq 20 \mathrm{mmol} / \mathrm{L}$ & $25(59.5 \%)$ & $13(34.2 \%)$ & 0.024 \\
APACHE II score $\geq 20$ & $31(73.8 \%)$ & $15(39.5 \%)$ & 0.002 \\
SOFA score $\geq 10$ & $24(57.1 \%)$ & $13(34.2 \%)$ & 0.040 \\
Septic shock & $25(59.5 \%)$ & $14(36.8 \%)$ & 0.043 \\
\hline
\end{tabular}

Data are presented as n (\%).

ICUAC: intensive care unit acquired candidemia, APACHE: acute physiology and chronic health evaluation,

SOFA: Sequential Organ Failure Assessment

Table 5. Univariate Analysis of Outcome in Patients with non-ICUAC.

\begin{tabular}{|c|c|c|c|}
\hline Characteristics & $\begin{array}{l}\text { Non-survived } \\
\quad(\mathrm{n}=54)\end{array}$ & $\begin{array}{r}\text { Survived } \\
(\mathrm{n}=93)\end{array}$ & $\mathrm{p}$ value \\
\hline Age $\geq 60$ years & $22(40.7)$ & $17(18.3)$ & 0.002 \\
\hline Solid tumor & $15(27.8)$ & $11(11.8)$ & 0.015 \\
\hline Hematological malignancy & $11(20.4)$ & $8(8.6)$ & 0.040 \\
\hline Neutropenia & $13(24.1)$ & $10(10.8)$ & 0.032 \\
\hline $\begin{array}{l}\text { Respiratory failure supported with non-invasive mechanical } \\
\text { ventilation }\end{array}$ & $12(22.2)$ & $7(7.5)$ & 0.010 \\
\hline CVC placement & $27(50.0)$ & $30(32.2)$ & 0.033 \\
\hline Concurrent bacteremia & $16(29.6)$ & $6(6.5)$ & 0.001 \\
\hline $\begin{array}{l}\text { Antifungal therapy started before microbiological } \\
\text { documentation }\end{array}$ & $17(31.5)$ & $47(50.5)$ & 0.025 \\
\hline Adequate antifungal therapy & $12(22.2)$ & $45(48.4)$ & 0.002 \\
\hline Absence of antifungal therapy & $14(25.9)$ & $11(11.8)$ & 0.028 \\
\hline Hemoglobin $\leq 70 \mathrm{~g} / \mathrm{L}$ & $19(35.2)$ & $18(19.4)$ & 0.033 \\
\hline Serum creatine $\geq 180 \mu \mathrm{mol} / \mathrm{L}$ & $7(13.0)$ & $3(3.2)$ & 0.050 \\
\hline APACHE II score $\geq 20$ & $21(38.9)$ & $15(16.1)$ & 0.002 \\
\hline SOFA score $\geq 10$ & $9(16.7)$ & $6(6.5)$ & 0.049 \\
\hline
\end{tabular}

tients with advanced age and concurrent bacteremia.

The APACHE II scoring system, which is widely used as a measure of the severity of illness and a predictor for death in ICU settings (20), is not often used in non-ICU settings. However, for the patients with candidemia, a higher APACHE II score was identified as an independent risk factor for death $(33,37)$. Our study highlights the prognostic significance of APACHE II in patients with candidemia in both ICU and non-ICU settings, indicating that the APACHE II score is a useful tool for the prediction of death in patients with candidemia admitted to non-ICU departments.

There are several limitations associated with our study. First, this study is an analysis of retrospectively gathered data, and may be subject to the limitations of the retrospective cohort design. Second, we did not further confirm the species of the strains genetically by deoxyribonucleic acid sequencing, which is reported to be more accurate than the VITEK-32 system used in this study. However, the results obtained using the VITEK-32 system that showed poor dis- crimination or non-identification at the level of species were not included, which would further elevate the accuracy of the system as applied to this study. Third, we did not collect data on the exact time between candidemic episodes and the initiation of antifungal therapy, which, in addition to the sample size, precluded further analysis of the effect of the time difference on the clinical outcome. Fourth, we were not able to evaluate the long-term prognosis (including 6-month or 1-year outcomes) due to the high incidence of loss to follow-up. Therefore, we were limited to day-90 mortality as the mortality outcome in this study.

In conclusion, the Candida species and antifungal resistance profiles in patients with ICUAC were similar to those of non-ICUAC patients, but led to worse outcomes. In both groups, higher APACHE II scores were shown to be a common predictor of death, and adequate antifungal therapy should be encouraged in order to decrease mortality. Additional predictors of death that were differentially associated with the ICUAC and non-ICUAC patient groups may be relevant for the clinical management of patients with can- 
didemia in ICU and non-ICU settings.

The authors state that they have no Conflict of Interest (COI).

\section{Financial Support}

Research was funded by the Shandong Provincial Natural Science Foundation of China (no. ZR2012HM005 and ZR2014HP 056).

\section{Acknowledgement}

We thank Hai-Ning Lu, Hai-Shang Wang, Lin-Na Tang and Jie Yang for data collection.

\section{References}

1. Horn DL, Neofytos D, Anaissie EJ, et al. Epidemiology and outcomes of candidemia in 2019 patients: data from the prospective antifungal therapy alliance registry. Clin Infect Dis 48: 1695-1703, 2009.

2. Ostrosky-Zeichner L, Kullberg BJ, Bow EJ, et al. Early treatment of candidemia in adults: a review. Med Mycol 49: 113-120, 2011.

3. Wisplinghoff H, Bischoff T, Tallent SM, Seifert H, Wenzel RP, Edmond MB. Nosocomial bloodstream infections in US hospitals: analysis of 24,179 cases from a prospective nationwide surveillance study. Clin Infect Dis 39: 309-317, 2004.

4. Marchetti O, Bille J, Fluckiger U, et al. Epidemiology of candidemia in Swiss tertiary care hospitals: secular trends, 1991-2000. Clin Infect Dis 38: 311-320, 2004.

5. Almirante B, Rodriguez D, Park BJ, et al. Epidemiology and predictors of mortality in cases of Candida bloodstream infection: results from population-based surveillance, barcelona, Spain, from 2002 to 2003. J Clin Microbiol 43: 1829-1835, 2005.

6. Bouza E, Munoz P. Epidemiology of candidemia in intensive care units. Int J Antimicrob Agents 32 (Suppl 2): S87-S91, 2008.

7. Pfaller M, Neofytos D, Diekema D, et al. Epidemiology and outcomes of candidemia in 3648 patients: data from the Prospective Antifungal Therapy (PATH Alliance(R)) registry, 2004-2008. Diagn Microbiol Infect Dis 74: 323-331, 2012.

8. Mikulska M, Del Bono V, Ratto S, et al. Occurrence, presentation and treatment of candidemia. Expert Rev Clin Immunol 8: 755765, 2012.

9. Pfaller MA, Diekema DJ. Epidemiology of invasive mycoses in North America. Crit Rev Microbiol 36: 1-53, 2010.

10. Bassetti M, Righi E, Costa A, et al. Epidemiological trends in nosocomial candidemia in intensive care. BMC Infect Dis 6: 21 , 2006.

11. Leroy O, Gangneux JP, Montravers P, et al. Epidemiology, management, and risk factors for death of invasive Candida infections in critical care: a multicenter, prospective, observational study in France (2005-2006). Crit Care Med 37: 1612-1618, 2009.

12. Zhang XB, Yu SJ, Yu JX, Gong YL, Feng W, Sun FJ. Retrospective analysis of epidemiology and prognostic factors for candidemia at a hospital in China, 2000-2009. Jpn J Infect Dis 65: 510515, 2012.

13. Playford EG, Nimmo GR, Tilse M, Sorrell TC. Increasing incidence of candidaemia: long-term epidemiological trends, Queensland, Australia, 1999-2008. J Hosp Infect 76: 46-51, 2010.

14. Davis SL, Vazquez JA, McKinnon PS. Epidemiology, risk factors, and outcomes of Candida albicans versus non-albicans candidemia in nonneutropenic patients. Ann Pharmacother 41: 568-573, 2007.

15. Aliyu SH, Enoch DA, Abubakar II, et al. Candidaemia in a large teaching hospital: a clinical audit. QJM 99: 655-663, 2006.
16. Uzun O, Ascioglu S, Anaissie EJ, Rex JH. Risk factors and predictors of outcome in patients with cancer and breakthrough candidemia. Clin Infect Dis 32: 1713-1717, 2001.

17. Colombo AL, Guimarães T, Silva LR, et al. Prospective observational study of candidemia in São Paulo, Brazil: incidence rate, epidemiology, and predictors of mortality. Infect Control Hosp Epidemiol 28: 570-576, 2007.

18. Viudes A, Pemán J, Cantón E, Ubeda P, López-Ribot JL, Gobernado M. Candidemia at a tertiary-care hospital: epidemiology, treatment, clinical outcome and risk factors for death. Eur $\mathbf{J}$ Clin Microbiol Infect Dis 21: 767-774, 2002.

19. Bassetti M, Trecarichi EM, Righi E, et al. Incidence, risk factors, and predictors of outcome of candidemia. Survey in 2 Italian university hospitals. Diagn Microbiol Infect Dis 58: 325-331, 2007.

20. Chang RW, Jacobs S, Lee B. Predicting outcome among intensive care unit patients using computerised trend analysis of daily Apache II scores corrected for organ system failure. Intensive Care Med 14: 558-566, 1988.

21. Vincent JL, de Mendonça A, Cantraine F, et al. Use of the SOFA score to assess the incidence of organ dysfunction/failure in intensive care units: results of a multicenter, prospective study. Working group on "sepsis-related problems" of the European Society of Intensive Care Medicine. Crit Care Med 26: 1793-1800, 1998.

22. Pfaller MA, Andes D, Diekema DJ, et al. Wild-type MIC distributions, epidemiological cutoff values and species-specific clinical breakpoints for fluconazole and Candida: time for harmonization of CLSI and EUCAST broth microdilution methods. Drug Resist Updat 13: 180-195, 2010.

23. Pfaller MA, Andes D, Arendrup MC, et al. Clinical breakpoints for voriconazole and Candida spp. revisited: review of microbiologic, molecular, pharmacodynamic, and clinical data as they pertain to the development of species-specific interpretive criteria. Diagn Microbiol Infect Dis 70: 330-343, 2011.

24. Pfaller MA, Diekema DJ, Andes D, et al. Clinical breakpoints for the echinocandins and Candida revisited: integration of molecular, clinical, and microbiological data to arrive at species-specific interpretive criteria. Drug Resist Updat 14: 164-176, 2011.

25. Clinical and Laboratory Standards Institute (CLSI). Reference method for broth dilution antifungal susceptibility testing of yeasts: Third informational supplement. Rex JH, Alexander BD, Andes D, et al, Eds. CLSI, Wayne, PA, 2008: 1-28.

26. Yang YL, Ho YA, Cheng HH, Ho M, Lo HJ. Susceptibilities of Candida species to amphotericin B and fluconazole: the emergence of fluconazole resistance in Candida tropicalis. Infect Control Hosp Epidemiol 25: 60-64, 2004.

27. Marik PE, Lipman J. The definition of septic shock: implications for treatment. Crit Care Resusc 9: 101-103, 2007.

28. Pappas PG, Kauffman CA, Andes D, et al. Clinical practice guidelines for the management of candidiasis: 2009 update by the Infectious Diseases Society of America. Clin Infect Dis 48: 503-535, 2009.

29. Rodloff C, Koch D, Schaumann R. Epidemiology and antifungal resistance in invasive candidiasis. Eur J Med Res 16: 187-195, 2011.

30. De Luca C, Guglielminetti M, Ferrario A, Calabr M, Casari E. Candidemia: species involved, virulence factors and antimycotic susceptibility. New Microbiol 35: 459-468, 2012.

31. Bassetti M, Molinari MP, Mussap M, Viscoli C, Righi E. Candidaemia in internal medicine departments: the burden of a rising problem. Clin Microbiol Infect 19: E281-E284, 2013.

32. Pfaller MA, Messer SA, Moet GJ, Jones RN, Castanheira M. Candida bloodstream infections: comparison of species distribution and resistance to echinocandin and azole antifungal agents in Intensive Care Unit (ICU) and non-ICU settings in the SENTRY Antimicrobial Surveillance Program (2008-2009). Int J Antimicrob 
Agents 38: 65-69, 2011.

33. Andes DR, Safdar N, Baddley JW, et al. Impact of treatment strategy on outcomes in patients with candidemia and other forms of invasive candidiasis: a patient-level quantitative review of randomized trials. Clin Infect Dis 54: 1110-1122, 2012.

34. Charles PE, Doise JM, Quenot JP, et al. Candidemia in critically ill patients: difference of outcome between medical and surgical patients. Intensive Care Med 29: 2162-2169, 2003.

35. Sipsas NV, Lewis RE, Tarrand J, et al. Candidemia in patients with hematologic malignancies in the era of new antifungal agents (2001-2007): stable incidence but changing epidemiology of a still frequently lethal infection. Cancer 115: 4745-4752, 2009.

36. Bougnoux ME, Kac G, Aegerter P, d'Enfert C, Fagon JY; CandiRea Study Group. Candidemia and candiduria in critically ill patients admitted to intensive care units in France: incidence, molecular diversity, management and outcome. Intensive Care Med 34: 292-299, 2008.

37. Garey KW, Rege M, Pai MP, et al. Time to initiation of flucona- zole therapy impacts mortality in patients with candidemia: a multi-institutional study. Clin Infect Dis 43: 25-31, 2006.

38. Zilberberg MD, Kollef MH, Arnold $\mathrm{H}$, et al. Inappropriate empiric antifungal therapy for candidemia in the ICU and hospital resource utilization: a retrospective cohort study. BMC Infect Dis 10: $150,2010$.

39. Garnacho-Montero J, Díaz-Martín A, García-Cabrera E, Ruiz Pérez de Pipaón M, Hernández-Caballero C, Lepe-Jiménez JA. Impact on hospital mortality of catheter removal and adequate antifungal therapy in Candida spp. bloodstream infections. J Antimicrob Chemother 68: 206-213, 2013.

40. Metnitz PG, Krenn CG, Steltzer H, et al. Effect of acute renal failure requiring renal replacement therapy on outcome in critically ill patients. Crit Care Med 30: 2051-2058, 2002.

41. Esteban A, Anzueto A, Frutos F, et al. Characteristics and outcomes in adult patients receiving mechanical ventilation: a 28-day international study. JAMA 287: 345-355, 2002.

(C) 2015 The Japanese Society of Internal Medicine http://www.naika.or.jp/imonline/index.html 